\title{
Azithromycin Oral Suspension in Prevention and Management of Oral Mucositis in Patients Undergoing Hematopoietic Stem Cell Transplantation: A Randomized Controlled Trial
}

\section{Sayeh Parkhideh}

Shahid Beheshti University of Medical Sciences School of Medicine

\section{Mohadeseh Zeraatkar}

Shahid Beheshti University of Medical Sciences School of Pharmacy

Omid Moradi

Shahid Beheshti University of Medical Sciences School of Pharmacy

Abbas Hajifathali

Shahid Beheshti University of Medical Sciences School of Medicine

Mahshid Mehdizadeh

Shahid Beheshti University of Medical Sciences School of Medicine

Maria Tavakoli Ardakani ( $\square$ mariatavakoli@yahoo.com )

Shahid Beheshti University of Medical Sciences School of Pharmacy

\section{Research Article}

Keywords: Azithromycin, Mucositis, Supportive care, Hematopoietic stem cell transplantation

Posted Date: June 7th, 2021

DOI: https://doi.org/10.21203/rs.3.rs-250206/v1

License: (c) (i) This work is licensed under a Creative Commons Attribution 4.0 International License. Read Full License

Version of Record: A version of this preprint was published at Supportive Care in Cancer on July 14th, 2021. See the published version at https://doi.org/10.1007/s00520-021-06409-0. 


\section{Abstract}

Objectives: This study aimed to investigate the effects of Azithromycin suspension on oral mucositis in patients undergoing hematopoietic stem cell transplantation (HSCT)

Methods and material: The study was designed as a single-blind randomized controlled trial in Taleghani medical center affiliated to Shahid Beheshti University of Medical Sciences Tehran Iran. Patients undergoing HSCT were randomly assigned to intervention or control groups. Azithromycin suspension was administered twice daily for gargling for 30 seconds and swallow, on the first day of chemotherapy for patients in the intervention group. Graded Oral Mucositis (OM) occurrence based on National Cancer Institute Common Toxicity Criteria (NCl-CTC) scale (grade 0 to 5 ) considered as the main outcome and the Numerical Rating Scale (NRS:0-10) measured the severity of OM symptoms.

Results: In a duration of fifteen months, 88 patients were randomly assigned and finally 70 patients were evaluable for study outcomes (randomized 1:1 to azithromycin versus no-azithromycin). The incidence and duration of the mucositis significantly improved in the intervention group compared to the control. Azithromycin use was consistent with a lower rate of dryness $(P<0.001)$, dysphagia $(P<0.001)$, and loss of sense of taste $(P<0.001)$. Also, in the intervention group, lower intensity of pain due to mucositis $(P=0.01)$ and lower duration of mucositis were observed $(p=0.045)$. No significant adverse drug reaction was observed in patients receiving azithromycin.

Conclusion: Based on the result from this study Azithromycin suspension is an effective option in the prevention and treatment of chemotherapy-induced OM. Further study is needed to assess the effect of azithromycin and comparison with other therapeutic options.

\section{Introduction}

Hematopoietic Stem Cell Transplantation (HSCT) is an acceptable cure in many hematologic and autoimmune diseases (1). This therapeutic approach involves harvesting, maintaining, and administering stem cells from a donor to the patient after using chemotherapy $(2,3)$. Donor and recipient are the same in autologous transplant but the donor is from related or unrelated human leukocyte antigen (HLA) matched in allogeneic transplantation (4). Using high doses of chemotherapy medication as a conditioning regimen is accompanied by serious adverse reactions (5). Mucositis is a dose-limiting complication of chemotherapy in HSCT patients. Mucosal injury is characterized by inflammation, pain, redness, edema, ulcer and bleeding, and superinfections (6). This dose-limiting ADR negatively affects the nutritional condition of the patients (7). Oral mucositis (OM) is seen in $40 \%$ of the chemotherapy cycles and $7 \%$ in gastric mucosa in cancer chemotherapy (8). Due to higher doses of chemotherapy agents in HSCT mucositis has been reported in about $70-80 \%$ of patients (9). Mostly, mucosal injury occurs $5-6$ days after cytotoxic therapy and reach their maximum in 14 days (6). Clinically, mucositis usually begins with erythema or pain and can cause the spread of ulcers and result in the problem of swallowing and reducing nutritional intake (10). In the case of severe thrombocytopenia, oral bleeding may occur in these patients (11). 
In order to decrease the symptoms associated with $\mathrm{OM}$, different agents have been investigated. Due to the inflammatory and microbial process of mucositis, many antibiotics and anti-inflammatory agents had been tested to control and prevent mucositis. In the process of mucositis, the most isolated gram-positive microorganisms are staphylococcus and streptococcus colonies (12). Azithromycin is a macrolide antibiotic with anti-inflammatory properties by accumulation in the polymorphonuclear leukocytes and macrophages, and modulate the inflammatory response $(13,14)$. In addition, azithromycin antimicrobial effects against Staphylococci and Streptococci species could be effective in the prevention and treatment of OM (15). Due to the anti-inflammatory, immunomodulatory and anti-microbial effects of azithromycin, we hypothesized that oral azithromycin suspension could be effective in preventing and treating mucositis and reducing the complications of mucositis.

\section{Methods And Materials}

\section{Study design and patient's selection}

We conducted a single-blind, randomized, controlled clinical trial from February 2016 to May 2017 at the bone marrow transplantation center, Taleghani Teaching Hospital affiliated to Shahid Beheshti University of Medical Sciences, Tehran, Iran. The study was conducted in accordance to the declaration of Helsinki and was approved by the board of ethics committee in Shahid Beheshti University of Medical Sciences and registered in the Iranian Registry of Clinical Trials (IRCT201603093210N13). Written informed consent was signed by all participants or their legal guardians before enrollment. The inclusion criteria were defined as followed. Patients aged 15 years and older, being a nonsmoker, able to gargle mouthwash solution, with no history of liver disease and capability of reading and communicating with staff and able to sign the informed consents included to the study.Exclusion criteria included any serious adverse effect of azithromycin or allergic reaction to azithromycin suspension, and failure to follow the instruction to use oral protocol due to any variation of the patient's health condition.All included patients were randomly assigned into two groups of azithromycin suspension and control. Randomization of patients was stratified with respect to age, gender, type of transplantation, and malignancy

\section{Intervention}

On the first day of hospitalization, patients are provided with instructions to maintain good oral hygiene. Based on bone marrow transplant (BMT) ward protocol, during the hospitalization time, patients in both groups received sodium chloride $0.9 \%$ and chlorhexidine mouthwash $5 \mathrm{~mL}$ three times daily to reduce the risk of oral infections.By adding $30 \mathrm{~mL}$ pre-boiled water to the bottle of Azithromycin (Tehran Cheimie Pharmaceutical Company, Tehran, Iran) powder up to the marked line, the suspension is ready to use by the concentration of $200 \mathrm{mg} / 5 \mathrm{~mL}$. The prepared medication should be consumed by the patients in 48 hours $7.5 \mathrm{~mL}$ twice daily. On the first day of chemotherapy patients in the test group, received Azithromycin suspension. They were requested to shake well and gargle $7.5 \mathrm{~mL}$ suspension for 30 seconds or more and then swallow twice daily. The therapy continued to the engraftment day or mucositis resolution.Oral examination was performed from the first day of chemotherapy before intervention and daily thereafter. To control and manage the symptoms of $\mathrm{OM}$ after the occurrence, chlorhexidine mouthwash discontinued and 
symptoms were managed using a mouthwash containing diphenhydramine $12.5 \mathrm{mg} / 5 \mathrm{~mL}$, lidocaine $2 \%$, aluminum hydroxide $225 \mathrm{mg} / 5 \mathrm{~mL}$, magnesium hydroxide $200 \mathrm{mg} / 5 \mathrm{~mL}$, and nystatin $100000 \mathrm{IU} / 12 \mathrm{~mL}$ was prescribed. Refractory episodes of pain were controlled using opioids as patient control analgesia (PCA) administration in both groups until engraftment day or recovery of the mucosa. Also, patients in the intervention group were instructed to use Azithromycin suspension until the engraftment day or resolution of OM. Moreover, the standard protocol of the BMT ward continued for managing mucositis.

\section{Assessment and outcome measures}

For each patient, basic demographics and clinical data including age, gender, type of malignancy, type of transplantation, and any history of allergy to drugs were recorded in prepared sheets. laboratory data consisting of White Blood Cell (WBC), platelet count, blood culture before and after the intervention, time of total parenteral nutrition (PN) initiation and its duration (if any), need to opioid analgesic and dose of it (if any), need to receive antibiotics and length of hospital stay were recorded until discharge from the ward.All patients were assessed for $\mathrm{OM}$ episode incidence as the primary outcome. The patients were visited by the same practitioner to be examined in accordance with the mucositis and the practitioner was blinded to the allocation. Patients who were not excluded from the study and finished the protocol were analyzed. The secondary outcomes measured in both groups as the time of mucositis occurrence, duration of OM episodes, maximum grade and, average daily degree of mucositis, the necessity to receive other mucositis therapies, need to receive and duration of PN, antibiotics utilization, length of hospital stay, days with fever, first episode of infection and engraftment time. Also, patients were assessed for severity of the symptoms and pain, dryness of oral cavity, dysphagia and alteration taste perception, serum glutamate-pyruvate transaminase (SGPT), and serum glutamic-oxaloacetic transaminase (SGOT) (due to azithromycin side effect), Serum creatinine, were assessed and recorded for each patient during the study period.Engraftment time was represented as the day on which the absolute neutrophil count was greater than 500 per microliter for 3 days in a row and platelet count of 20000 per microliter for 3 consecutive days (16). Infection was considered as a positive blood culture and fever was defined as a single oral temperature equal, or more than a based on National Comprehensive Cancer Network (NCCN) guideline.National Cancer Institute Common Toxicity Criteria (NCI-CTC) version 4 mucositis scale was used to measure the OM severity (Table 1) (17). In patients developing $O M$, the Oral Mucositis Daily Assessment Worksheet was completed and the severity of symptoms was evaluated using the Numerical Rating Scale (NRS: 0-10).

\section{Statistical analysis}

Intention-to-treat analysis was considered. In the case of quantitative variables, the results were calculated as the mean \pm standard deviation, and in the nominal and qualitative variables, the results were expressed as percentages. To compare quantitative variables with normal distribution, we used paired and unpaired ttest, and to compare quantitative variables with non-normal distribution, use Mann-U-Whitney test. Chisquare was used to compare qualitative and nominal variables. Whenever the chi-square test was more than $25 \%$ of the expected values less than 5, we used Fisher Exact test instead of the chi-square test. All tests were of two-tailed type. The normal distribution of the variables was evaluated using the KolmogorovSmirnov test. P-value less than 0.05 was considered statistically significant. Statistical analysis was 
performed using SPSS Version 22 software and Minitab software. The 2-sided p-value less than 0.05 is considered as significant.

\section{Results}

A total of 88 eligible patients who met the inclusion criteria were enrolled in this study. Of these, 41 patients were assigned to the treatment group and 47 patients were assigned to the control group. Out of 88 enrolled patients, 18 patients were excluded due to prolonged neutropenia phase, death, variation of patients' health status, gastrointestinal complications following transplantation, and lack of cooperation. The detailed study diagram is expressed in Figure 1.Table 2 demonstrated the demographics and basic characteristics of 70 patients who successfully continued the study. No significant difference was observed in gender, age, type of transplantation, and malignancy between groups.Developing OM significantly decreased in the treatment group $(P=0.015)$. OM occurred in $12(43.3 \%)$ patients who received azithromycin compared to $22(62.9 \%)$ in the control group. In total 34 patients diagnosed with $\mathrm{OM}$, the mean time of onset was $6.1 \pm 2.2$ days and azithromycin was associated with delayed onset $(7.5 \pm 1.4 \mathrm{vs}$ $5.3 \pm 2.2, P=0.015)$. Furthermore, in the treatment group, the duration of mucositis was $5.1 \pm 1.3$ days in comparison to $8.8 \pm 4.1$ days in control patients which were statistically significant $(p=0.045)$. But there was no significant difference in the maximum grade of mucositis $(p=0.157)$ and average daily grade of mucositis $(p=0.298)$.In terms of OM symptoms, a significant decrease was detected in the level of pain $(p=0.01)$, dryness of oral cavity $(p<0.001)$, sense of taste $(p<0.001)$, and dysphagia $(p<0.001)$ in the azithromycin suspension group. Compared with the control group, the frequency and description of OM are represented in Table 3.We found that patients receiving Azithromycin suspension were less likely to require other OM remedies. Ten patients in the intervention and 22 patients in the control group received the treatment $(p=0.004)$ and the duration of need for other remedies in the azithromycin group was $1.5 \pm 2.6$ and 5.2 \pm 5.1 days in the control group $(p=0.05)$. Patients who received azithromycin needed lower narcotic analgesics administration ( $6 \mathrm{vs} 18, p=0.003)$ and the total dose of morphine sulfate decreased $(45.8 \pm 24.6$ vs $179.2 \pm 23.7, p<0.001)$ in the treatment group compared to the control group. In addition, $7(20 \%)$ patients of the treatment group vs $16(45.7 \%)$ of the control group patients needed to receive parenteral nutrition support ( $p=0.041)$ with the duration of $3.1 \pm 1.1$ vs $3.2 \pm 1.3$ days in intervention and control groups, respectively. The duration of days of PN in treatment and control groups were $0.7 \pm 2.0$ and $3.8 \pm 7.7$ days, respectively, and was not significantly different $(p=0.922)$. However, the duration of hospitalization did not change significantly between the two groups $(p=0.814)$ (treatment group: $27.5 \pm 4.7$ days, control group: $28.5 \pm 6.4$ days).

Fever as an indicator of infection was recorded for $1.5 \pm 3.6$ days in patients who received azithromycin and $0.9 \pm 1.6$ days in the control group. The difference was not statistically significant $(p=0.68)$. The time of platelet and WBC engraftment was not statistically different between the two groups. The average use of antibiotics in the treatment group was $1.4 \pm 1.3$ and $1.9 \pm 1.5$ in the control group. No significant difference in the use of antibiotics was found between the two groups ( $p=0.088)$. In accordance with positive blood culture, two patients in the intervention and seven patients in the control group found positive ( $P=0.047)$ that was significant. 
By considering safety outcomes, adverse drug reactions associated with azithromycin use, and adherence to the therapy, only 2 patients have failed to receive the medication due to signs and symptoms of gastrointestinal disorders (consisting of diarrhea, nausea and vomiting). The QTc prolongation did not occur in the course of therapy in patients who received cardiac monitoring.

\section{Discussion}

OM refers to erythematous and ulcerative lesions of the oral cavity mucosa, is observed in patients with cancer who received chemotherapy, and/or with radiation therapy to fields involving the oral cavity (6). Previously, many treatment options have been proposed to alleviate the bothersome symptoms of these phenomena (18). In the present study, by administrating azithromycin in mouthwash dosage form we delivered a high concentration of the medication to mucosa directly. The immunomodulatory and also antiinfective effect of azithromycin delivered to oral mucosa resulted in positive clinical outcomes such as less frequent mucositis episodes, and a decrease in associated symptoms severity. Also, patients who received azithromycin needed less parenteral nutrition support because of lower mucositis incidence and intensity, and also the functioning gastrointestinal tract in this population have important medical and psychological aspects. Yuen $\mathrm{KY}$ et.al evaluated the effect of Clarithromycin on mucositis in 70 patients undergoing bone marrow transplantation in 2001. Clarithromycin was prescribed $500 \mathrm{mg}$ two times daily orally from 7 days before transplantation to 2 days before it then followed by $250 \mathrm{mg}$ clarithromycin two times daily until engraftment. Clarithromycin significantly decreased the incidence of OM compared to the control group. However, there was no significant difference between the two groups in terms of infection rate, duration of fever and need to PN, need for growth factors, incidence, and severity of graft versus host disease (GVHD) between the two groups could not be seen (19). Other therapies with immunomodulatory effects such as herbal medicine also showed positive effects in this population. Matricaria recutita and Mentha Piperita herbal mouthwash effects on the management of $\mathrm{OM}$ in patients undergoing hematopoietic stem cell transplantation were examined. The duration, maximum and average daily grade of $\mathrm{OM}$ were significantly reduced in the treatment group. compared to the control group. The use of herbal mouthwash led to significant improvements in pain intensity, dryness, and dysphagia. Other significant results include reduced need for complementary medications, narcotic analgesics, total parenteral nutrition (TPN) and the duration of PN have been observed(20).

Furthermore, azithromycin mouthwash administration was consistent with other clinical benefits along with reducing the incidence of mucositis. Although azithromycin was not associated with a decrease in the severity of the $\mathrm{OM}$ episodes according to the results, the time of mucositis was delayed approximately two days and the duration of the mucositis period decreased. Based on its effect on the immune system and protecting the damaged mucosa from superinfections, it seems tissue regeneration mucosa healing is accelerated, and alongside lower incidence, delayed episodes with decrease duration were observed. Also, we should emphasize the important effect of azithromycin on OM complications. As an important aspect of chemotherapy-induced OM, many of the patients are afraid of its complications such as dry mouth, taste perception problems. As important finding azithromycin significantly decreases the incidence of these complications. 
Other therapeutic agents with anti-inflammatory effects and antioxidants such as stimulating factors and nutritional supplementation have been examined. Hosseinjani et al in 2017 evaluate the efficacy of erythropoietin mouthwash in the prevention of OM in patients undergoing autologous HSCT. Based on its anti-inflammatory properties the results from this study indicated a clinically meaningful positive effect on the incidence and duration of OM (5). The efficacy of selenium was evaluated in the prevention of OM in patients undergoing HSCT as an antioxidant. Thirty-seven patients received oral selenium tablets ( $200 \mathrm{mcg}$ twice daily) from the starting day of high dose chemotherapy to 14 days after transplantation. The incidence of severe OM (grades 3-4) was significantly lower in the selenium group. Significant elevations in serum selenium level and plasma Glu.Px activity was observed 7 and 14 days after transplantation compared with baseline in the selenium group. They conclude that selenium. It could reduce the duration and severity of OM after high-dose chemotherapy (21). But we should consider limitations to use these therapies. The first and most important limitation to use stimulating factors such as erythropoietin is financial considerations. Compared to azithromycin suspension, erythropoietin is associated with a significantly higher price and the availability of the dosage form is also problematic.

Although azithromycin could be associated with some adverse reaction but based on the results, we did not record any serious adverse effects due to azithromycin suspension administration. But we still encourage to assess and monitor patients. For interpretation of the results, the limitations of the study should be considered. First, this study is not placebo-controlled. Second, the study is designed as a single-centered pilot study, and larger studies with more sample size are needed.

\section{Conclusions}

Azithromycin suspension can be effective in decreasing the incidence and duration of OM in patients who received HSCT. Also, the need for $\mathrm{PN}$ and duration of $\mathrm{PN}$ in this and analgesic therapy decreased. Based on its immunomodulatory and anti-infective properties and affordability and availability, azithromycin suspension is a reasonable option to use as a preventive and add-on therapy agent in a patient undergoing HSCT.

\section{Declarations}

\section{Funding:}

The study was conducted under the supervision of deputy of research and technology, Shahid Beheshti University of Medical Sciences, Tehran, Iran and did not receive any extra source of financial support from secondary institution or company.

Conflicts of interest/Competing interests:

The authors declare none.

Availability of data and material: 
The data that support the findings of this study are available on request from the corresponding author, [MTA]

Code availability:

N/A

Authors' contributions:

SP, Acquisition of data, Critical revision of the manuscript for important intellectual content, Study supervision; MZ, Acquisition of data, Drafting of the manuscript, Technical support; OM, Drafting of the manuscript, Statistical analysis, Critical revision of the manuscript for important intellectual content; $\mathrm{AH}$, Acquisition of data, Critical revision of the manuscript for important intellectual content, Study supervision; MM, Acquisition of data, Critical revision of the manuscript for important intellectual content, Study supervision; MTA, Study concept and design, Acquisition of data, Drafting of the manuscript, Statistical analysis, Administrative and material support, Critical revision of the manuscript for important intellectual content, Study supervision.

Ethics approval:

The study was approved by the board of ethics committee, Shahid Beheshti University of Medical Sciences, Tehran, Iran (Code: IR.SBMU.PHNM.1394.307)

Consent to participate:

The written informed consent was obtained from all patients prior to enrollment.

Consent for publication:

The consent for publication of the result of the study was obtained from all of the participants. No personal/contact information of the participant was reported in this study.

\section{References}

1. Barriga F, Ramírez P, Wietstruck A, Rojas N. Hematopoietic stem cell transplantation: clinical use and perspectives. Biological research. 2012;45(3):307-16.

2. Hölig K, Kramer M, Kroschinsky F, Bornhäuser M, Mengling T, Schmidt AH, et al. Safety and efficacy of hematopoietic stem cell collection from mobilized peripheral blood in unrelated volunteers: 12 years of single-center experience in 3928 donors. Blood. 2009;114(18):3757-63.

3. Suárez-Álvarez B, López-Vázquez A, López-Larrea C. Mobilization and homing of hematopoietic stem cells. Stem cell transplantation: Springer; 2012. p. 152-70.

4. Chen S-H, Wang T-F, Yang K-L. Hematopoietic stem cell donation. International journal of hematology. 2013;97(4):446-55. 
5. Hosseinjani H, Hadjibabaie M, Gholami K, Javadi M, Radfar M, Jahangard-Rafsanjani Z, et al. The efficacy of erythropoietin mouthwash in prevention of oral mucositis in patients undergoing autologous hematopoietic SCT: a double-blind, randomized, placebo-controlled trial. Hematological oncology. 2017;35(1):106-12.

6. Pulito C, Cristaudo A, Porta C, Zapperi S, Blandino G, Morrone A, et al. Oral mucositis: the hidden side of cancer therapy. Journal of experimental \& clinical cancer research : CR. 2020;39(1):210.

7. Lalla RV, Sonis ST, Peterson DE. Management of oral mucositis in patients who have cancer. Dental Clinics of North America. 2008;52(1):61-77.

8. Mercadante S, Aielli F, Adile C, Ferrera P, Valle A, Fusco F, et al. Prevalence of oral mucositis, dry mouth, and dysphagia in advanced cancer patients. 2015;23(11):3249-55.

9. Vagliano L, Feraut C, Gobetto G, Trunfio A, Errico A, Campani V, et al. Incidence and severity of oral mucositis in patients undergoing haematopoietic SCT-results of a multicentre study. 2011;46(5):72732.

10. Köstler WJ, Hejna M, Wenzel C, Zielinski CC. Oral mucositis complicating chemotherapy and/or radiotherapy: options for prevention and treatment. CA: a cancer journal for clinicians. 2001;51(5):290315.

11. Sonis ST. The pathobiology of mucositis. Nature Reviews Cancer. 2004;4(4):277-84.

12. Vanhoecke B, De Ryck T, Stringer A, Van de Wiele T, Keefe D. Microbiota and their role in the pathogenesis of oral mucositis. Oral diseases. 2015;21(1):17-30.

13. Zimmermann P, Ziesenitz VC, Curtis N, Ritz N. The immunomodulatory effects of macrolides-a systematic review of the underlying mechanisms. Frontiers in immunology. 2018;9:302.

14. Nozoe K, Aida Y, Fukuda T, Sanui T, Nishimura F. Mechanisms of the macrolide-induced inhibition of superoxide generation by neutrophils. Inflammation. 2016;39(3):1039-48.

15. Williams JD. Spectrum of activity of azithromycin. European journal of clinical microbiology \& infectious diseases : official publication of the European Society of Clinical Microbiology. 1991;10(10):813-20.

16. Saad A, de Lima M, Anand S, Bhatt VR, Bookout R, Chen G, et al. Hematopoietic Cell Transplantation, Version 2.2020, NCCN Clinical Practice Guidelines in Oncology. 2020;18(5):599-634.

17. Chen AP, Setser A, Anadkat MJ, Cotliar J, Olsen EA, Garden BC, et al. Grading dermatologic adverse events of cancer treatments: the Common Terminology Criteria for Adverse Events Version 4.0. 2012;67(5):1025-39.

18. Shankar A, Roy S, Bhandari M, Rath G, Biswas AS, Kanodia R, et al. Current trends in management of oral mucositis in cancer treatment. 2017;18(8):2019.

19. Yuen KY, Woo P, Tai J, Lie A, Luk J, Liang R. Effects of clarithromycin on oral mucositis in bone marrow transplant recipients. haematologica. 2001;86(5):554-5.

20. Ardakani MT, Ghassemi S, Mehdizadeh M, Mojab F, Salamzadeh J, Ghassemi S, et al. Evaluating the effect of Matricaria recutita and Mentha piperita herbal mouthwash on management of oral mucositis 
in patients undergoing hematopoietic stem cell transplantation: A randomized, double blind, placebo controlled clinical trial. Complementary therapies in medicine. 2016;29:29-34.

21. Jahangard-Rafsanjani Z, Gholami K, Hadjibabaie M, Shamshiri A, Alimoghadam K, Sarayani A, et al. The efficacy of selenium in prevention of oral mucositis in patients undergoing hematopoietic SCT: a randomized clinical trial. Bone Marrow Transplantation. 2013;48(6):832-6.

\section{Tables}

Table 1. National Cancer Institute Common Toxicity Criteria of mucositis

\begin{tabular}{|l|l|l|}
\hline Grade & Description \\
\hline $\begin{array}{l}\text { Grade } \\
0\end{array}$ & None \\
\hline $\begin{array}{l}\text { Grade } \\
1\end{array}$ & Painless ulcers, erythema, or mild soreness in the absence of lesions \\
\hline $\begin{array}{l}\text { Grade } \\
2\end{array}$ & Painful erythema, edema, or ulcers but eating or swallowing possible \\
\hline $\begin{array}{l}\text { Grade } \\
3\end{array}$ & Painful erythema, edema, or ulcers requiring IV hydration & \\
\hline $\begin{array}{l}\text { Grade } \\
4\end{array}$ & $\begin{array}{l}\text { Severe ulceration or requiring parenteral or enteral nutritional support or } \\
\text { prophylactic intubation }\end{array}$ & \\
\hline
\end{tabular}

Table 2. Patient's Demographics Characteristics and Related Clinical Data 


\begin{tabular}{|c|c|c|c|}
\hline Variable & $\begin{array}{l}\text { Intervention group } \\
\qquad(\mathrm{n}=35)\end{array}$ & Control group $(n=35)$ & $\mathrm{P}$ value \\
\hline $\begin{array}{l}\text { Age (mean } \pm \text { SD) } \\
\text { Years }\end{array}$ & $39.1 \pm 13.5$ & $44.1 \pm 13$ & 0.495 \\
\hline \multicolumn{4}{|l|}{ Gender (8) } \\
\hline $\begin{array}{l}\text { Female } \\
\text { Male }\end{array}$ & $\begin{array}{l}15(42.9) \\
20(57.1)\end{array}$ & $\begin{array}{l}17(48.6) \\
18(51.4)\end{array}$ & 0.405 \\
\hline \multicolumn{4}{|c|}{ Type of transplantation (8) } \\
\hline $\begin{array}{l}\text { Autologous } \\
\text { Allogeneic }\end{array}$ & $\begin{array}{l}25(71.4) \\
10(28.6)\end{array}$ & $\begin{array}{c}30(85.7) \\
5(14.3)\end{array}$ & 0.122 \\
\hline \multicolumn{4}{|l|}{ Type of malignancy } \\
\hline $\begin{array}{l}\text { Multiple myeloma } \\
\text { Lymphoma } \\
\text { Leukemia } \\
\text { Giant Cell Tumor }\end{array}$ & $\begin{array}{c}11(31.4) \\
15(42.9) \\
7(20) \\
2(5.7)\end{array}$ & $\begin{array}{c}13(37.1) \\
13(37.1) \\
7(20) \\
2(5.7)\end{array}$ & 0.997 \\
\hline
\end{tabular}

Table 3. Oral mucositis associated characteristics 


\begin{tabular}{|c|c|c|c|c|}
\hline \multicolumn{2}{|c|}{ OM characteristics } & $\begin{array}{c}\text { Intervention } \\
\text { group } \\
(\mathrm{n}=35)\end{array}$ & Control group $(\mathrm{n}=35)$ & $P$ value \\
\hline \multicolumn{2}{|c|}{ Frequency (8) } & $12(34.3)$ & $22(62.9)$ & 0.015 \\
\hline \multicolumn{2}{|c|}{ Onset (days) } & $7.5 \pm 1.4$ & $5.3 \pm 2.2$ & 0.015 \\
\hline \multicolumn{2}{|c|}{ Duration (days) } & $5.1 \pm 1.3$ & $8.8 \pm 4.1$ & 0.045 \\
\hline \multicolumn{2}{|l|}{ Grade } & $1.1 \pm 0.2$ & $1.3 \pm 0.3$ & 0.298 \\
\hline \multirow[t]{4}{*}{ Symptoms } & Pain & $2.0 \pm 0.7$ & $4.3 \pm 1.4$ & 0.010 \\
\hline & Dryness & $1.9 \pm 1.6$ & $3.8 \pm 1.9$ & $<0.001$ \\
\hline & Sense of taste & $8.9 \pm 1.1$ & $7.9 \pm 1.5$ & $<0.001$ \\
\hline & Dysphagia & $1.7 \pm 0.7$ & $2.6 \pm 2.6$ & $<0.001$ \\
\hline
\end{tabular}

Figures 


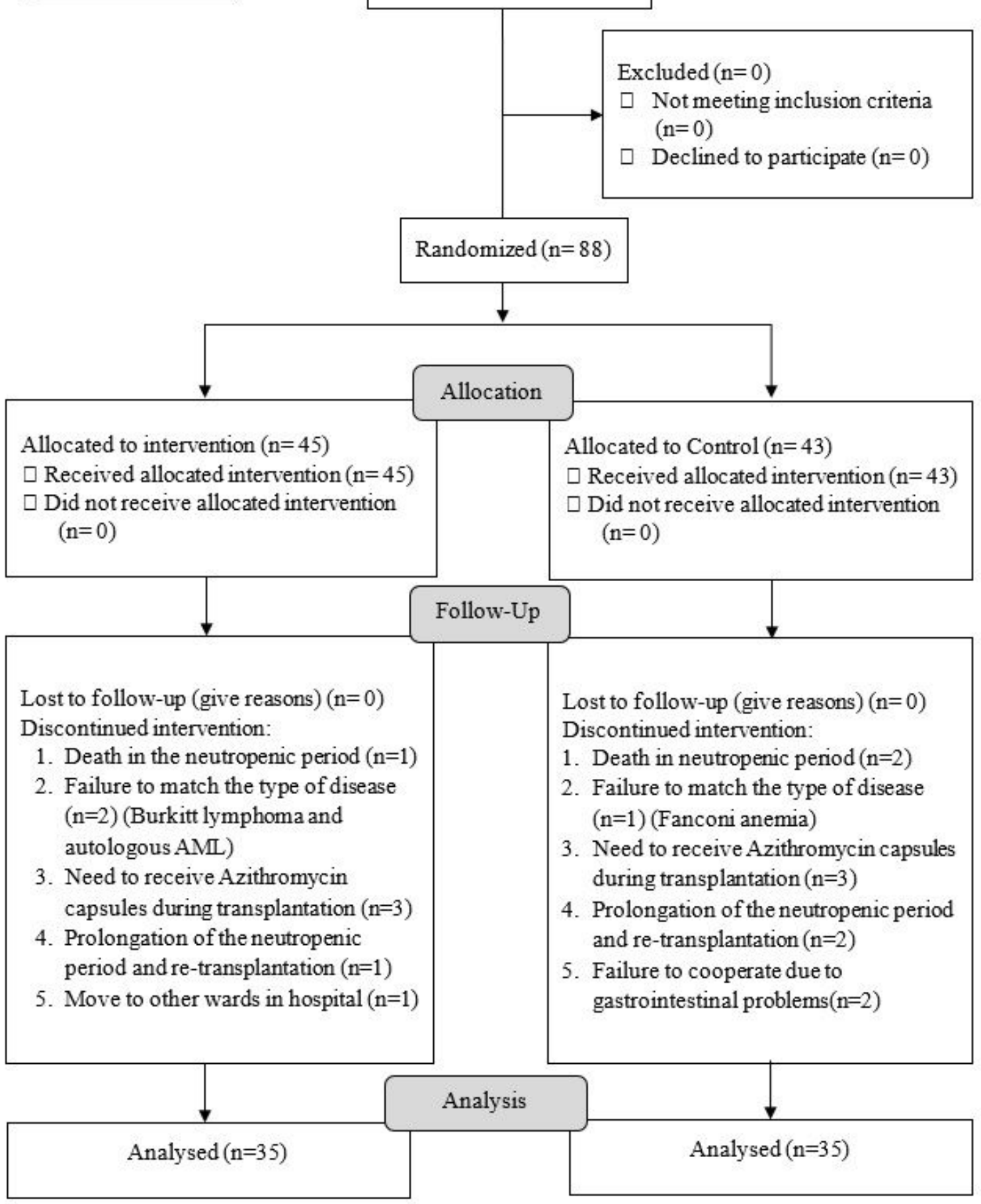

\section{Figure 1}

Flow diagram of the study 